\title{
Quality of life in extended endonasal approaches for skull
} base tumours*

C. Georgalas, R. Badloe, W. van Furth, S. Reinartz, W.J. Fokkens

Endoscopic Skull Base Center, Academic Medical Center, Amsterdam, The Netherlands
Rhinology 50: 255-261, 2012

DOI:10.4193/Rhino12.050

*Received for publication:

March 26, 2012

Accepted: May 7, 2012

\section{Summary}

Objective: To assess the quality of life (QOL) impact of extended endonasal approaches and nasoseptal flap reconstruction for benign skull base tumours

Methods: A random sample of 110 patients undergoing either limited endonasal transphenoidal hypophysectomy or extended endonasal approaches (trans-cribriform, trans-sellar, trans-tuberculum, trans-pterygoid) for the removal of benign skull base tumours were asked to complete Rhinosinusitis Outcome Measure (RSOM-31) questionnaire.

Results: A total of 91 patients returned the completed questionnaire. All patients in the limited approach group had pituitary adenomas, while patients in the extended group had a variety of tumours including adenomas with suprasellar or cavernous extension, chordomas, meningiomas, craniopharyngiomas and dermoids. Median time to completion of questionnaire was 1104 days in the limited group and 142 days in the extended approaches group. Although smell and headache were significantly worse in the group undergoing reconstruction with Haddad flap, there was no significant difference in overall, nasal, general, emotional or sleep quality of life between the two groups. Both smell and headache showed significant improvement with time. In linear regression, the single most important factor independently associated with overall worse RSOM-31 total scores was the presence of secreting adenomas.

Conclusion: The use of nasoseptal flap appears to have a limited negative impact in nasal quality of life, mainly related to headache and reduced smell, both of which tend to improve with time. Hormone-secreting tumours have the most important adverse effect in quality of life extending in general, emotional, sleep and overall wellbeing, as reflected in RSOM 31 subscales.

Keywords: Quality of life, Rhinosinusitis Outcome Measure (RSOM-31), pituitary surgery, skull base surgery, endoscopic transphenoidal hypophysectomy, endoscopic skull base surgery, meningioma, chordoma, craniopharyngioma, pituitary adenoma, dermoid tumour

\section{Introduction}

Extended endonasal approaches (EEA) are being increasingly applied over the last decade for the surgical management of benign and malignant tumours of the nose, paranasal sinuses and skull base ${ }^{(1,2)}$. Improved instrumentation, visualization and better understanding of the anatomy ${ }^{(3)}$, as well as the introduction of ' 4 hand, 2 nostril technique' (4) have all contributed to their success. However, a factor limiting their adoption has always been the extensive skull base defects created with the associated difficult to treat cerebrospinal fluid (CSF) leaks. A variety of techniques has been described for their management ${ }^{(5,6)}$; arguably, the most innovative and widely adopted has been the vascularized nasoseptal or Haddad-Bassagaisteguy flap. This is a mucoperichondrial pedicled flap of the septum with the septal branch of the sphenopalatine artery as its axial artery ${ }^{(7,8)}$. It is fair to say that the introduction of 
this relatively easy to raise, effective and reliable way to close skull base defects led to further adoption of such techniques and contributed to their popularity. However, harvesting this flap requires denuding of the septal mucerichondrium while adequate access predisposes extensive skull base drilling. Both of these factors are potentially associated with significant nasal morbidity, a fact that has not received adequate attention. We planned this study to assess the nasal and overall quality of life burden of such approaches as compared with traditional, less extensive, single nostril endoscopic transphenoidal pituitary surgery.

\section{Material and methods}

\section{Setting}

Tertiary academic Centre, subspecialized referral center (Endoscopic Skull base Amsterdam - Academic Medical Centre, University of Amsterdam).

\section{Study type}

Patient outcome study.

\section{Patients}

A random sample of 110 patients who underwent standard transphenoidal pituitary surgery or extended endonasal approaches to the ventral skull base in Academic Medical Centre between 2000 and 2010 were asked to complete Rhinosinusitis Outcome Measure (RSOM-31).

Pathology, approach used, operation date and patient characteristics were recorded from the patient's digital records.

\section{Outcome measures}

Primary outcome measure: Total score RSOM 31.

Secondary outcome measures: Nasal RSOM 31 summary score. General, Emotional, Sleep-related, Eye and Ear related RSOM 31 summary scores.

RSOM individual question scores.

\section{Statistical analysis}

Data was analyzed in PASW Statistics 18 (IBM Corporation, Sommers, NY, USA). Chi square and Fisher's exact test were used for comparing categorical variables, while comparisons between groups were performed using unpaired t test for normally distributed variables and Wilcoxon rank test for non parametric variables, as required. The level of significance was set at 0.05 for double - sided comparisons and Bonferroni correction was used for all multiple comparisons. Linear stepwise regression ( 0.10 entering, 0.05 removing variable threshold) was performed for assessing the predictor variables of the main outcome (RSOM 31 total score). Mean and SD were used to describe normally distributed variables and median and range non parametric variables. Although the distribution of total RSOM 31 score was normal, the distribution of individual questions as well of the subset summary measures was non parametric.

\section{Results}

\section{Patients}

A total of 91 patients completed the questionnaire (reply rate $83 \%)$. Sixty-four patients underwent standard endoscopic transphenoidal pituitary surgery and 27 underwent extended endonasal approaches with nasoseptal flap reconstruction. Their mean age was 53.5 years (SD 17.8, range 10 - 85) and 47 $(52 \%)$ were male. There was no significant difference in age or gender distribution between the two groups. The median time between surgery and completion of the questionnaire was 1104 days (range 64 - 3441 days) for the standard transphenoidal hypophysectomy group and 142 days (range 60 $577)$ for the extended approaches group ( $<<0.001)$.

\section{Pathologies}

Pathologies included hypophyseal adenomas (secreting and non-secreting, micro and macro adenomas), skull base (olfactory, tuberculum sellae) meningiomas, retrochiasmatic craniopharyngiomas and chordomas as well as Rathke cysts, dermoids and posterior skull base chordomas (Table 1).

Of the 27 secreting adenomas, 15 were GH producing, 6 were prolactinomas, 5 produced ACTH and $1 \mathrm{FSH}$. Regarding their anatomic extension, 77 (84.6\%) had suprasellar extension and $36(40 \%)$ were associated with cavernous sinus involvement, while 25 (27\%) were closely related to the carotid artery.

\section{Surgical technique}

Extended endonasal approaches were used for tumour extending beyond the sella - transcribriform for tumours of the olfactory fossa, transtuberculum and transphenoid for suprasellar/tuberculum sellae tumours, transpterygoid and transclivus for parasellar and posterior fossa tumours respectively. We used nasoseptal flap as the sole way of reconstructing initially, however with time our reconstructing technique evolved: We used a single, small piece of fat in order to fill the cavity taking care to avoid pressure to sensitive structures and applied fascia lata as inlay and the nasoseptal flap as overlay. We used tissue glue (Tissucol ${ }^{\oplus}$, Baxter, Utrecht, the Netherlands) and Spongostan ${ }^{\circledast}$ Absorbable Haemostatic Gelatin Sponge (Ethicon Biosurgery, Atlanta, GA, USA) over the nasoseptal flap. We used initially inflated balloon catheters for support, but we found subsequently that antibiotic-impregnated Vaseline gauze were more dependable. An intranasal splint was inserted to the flap side of the nasal septum and was removed after 3 weeks. Patients were instructed to gently rinse their nose with $10-20 \mathrm{ml}$ of normal saline for a minimum of 3 months after surgery and to avoid coughing and straining for the first 6 weeks postoperatively. 

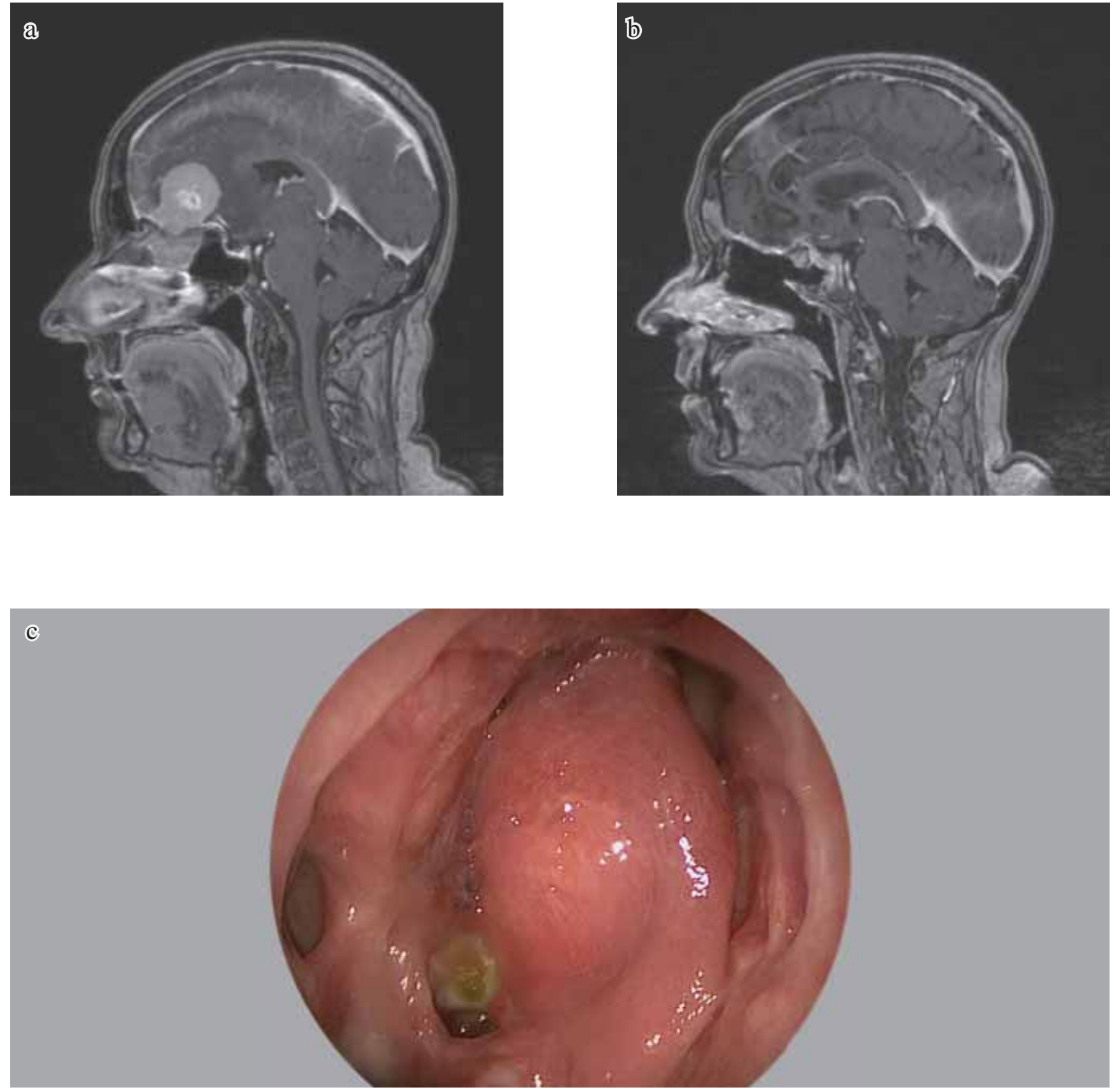

Figure 1a, b, c. Preoperative and postoperative scan of an olfactory crest meningioma and the endoscopic view of the nasoseptal flap 6 months after reconstruction.

\section{Questionnaire}

RSOM is divided in 5 categories: Nasal QOL (questions 1 - 6 nasal obstruction, rhinorrhea, sneezing, huposmia/hypogeusia, postnasal discharge, crusting), Eye QOL (questions 7 - 8), Sleep QOL (questions 9 - 12), Ear QOL (questions 13 - 17), General QOL (18 - 24, fatigue, reduced productivity, poor concentration, headache, cough, shortness of breath), Practical Issues (25 - 28, need to carry tissues/rub nose, blow nose, bad breath) and Emotional well being (29 - 31, feelings of frustration, helplessness, depression, irritability or embarrassment). We used RSOM 31 rather than its condensed and easier to administer form, SNOT 22, as we wanted to record patients overall quality of life, above and beyond their nasal symptoms.

The results for the questionnaire are seen in Figure 1 and Table 2 (from 0 to 5, better to worse QOL): there was no significant difference between the two groups, with the exception of Q 4 (hyposmia/hypogeusia: mean 1.03 median 0 standard group 


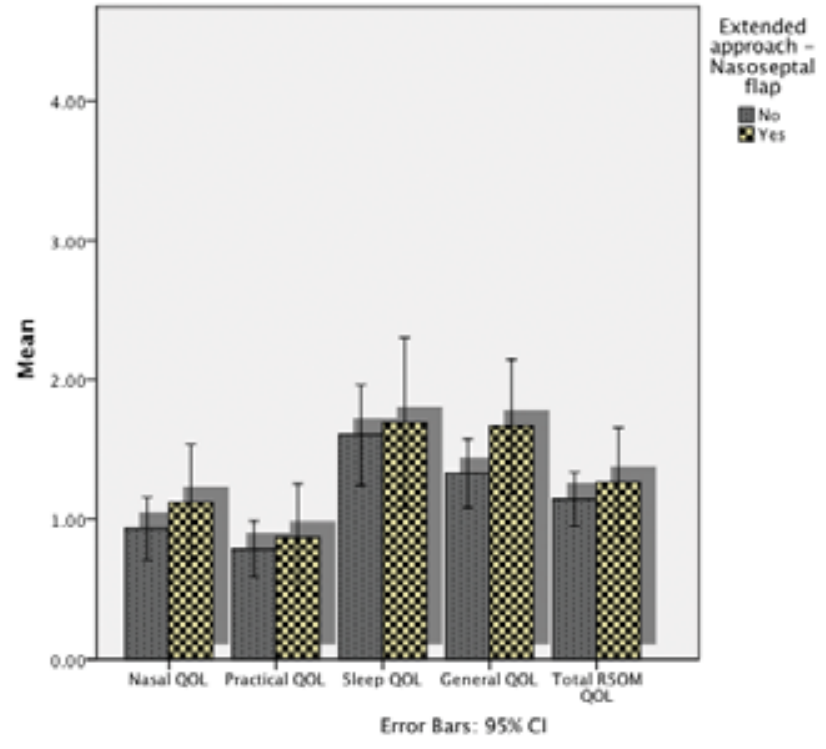

Figure 2. Mean RSOM subscales scores for patients undergoing extended approaches with the use of the nasoseptal flap compared with those undergoing standard endoscopic hypophysectomy.

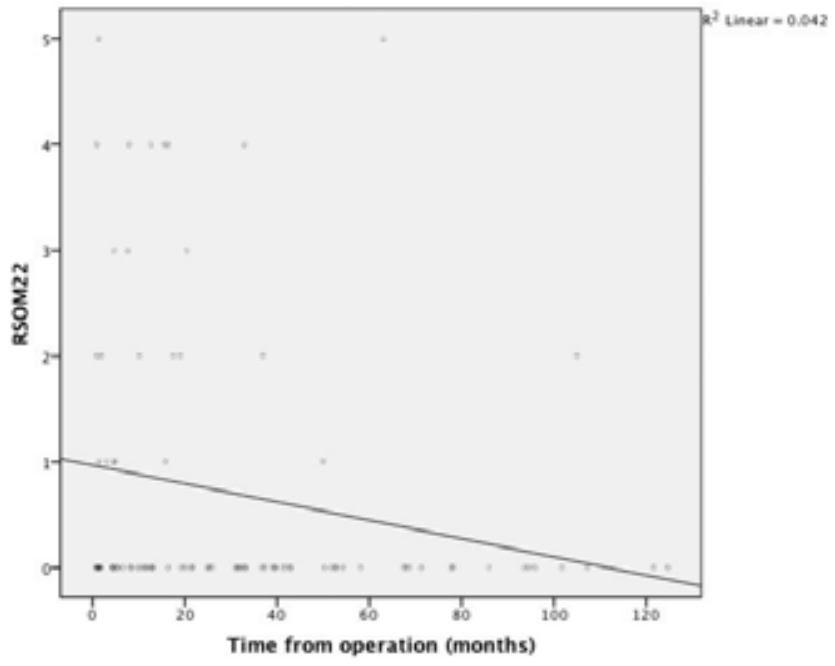

Figure 3. Evolution of headache with time from operation.
- mean 2.04 median 2 extended approach group, $\mathrm{p}=0.021$ ) and Q22 (Headache) mean 0.48 median 0 standard approach group, mean 1.19 median 0 extended approach group: $\mathrm{p}=$ 0.012. Total RSOM scores as well as general, emotional, nasal, practical and eye and ear related $\mathrm{QOL}$ were not statistically different between the two groups, although they tended to be worse in the extended approach group. Mean Total scores were 1.26 in the extended and 1.14 in the standard hypophysectomy group, while mean Nasal scores were 1.12 and 0.92 respectively. These were significantly lower than those seen in patients with CRS (typically higher than 2 and 2.5, total and nasal scores respectively). No significant differences were found between the different types of extended approaches in terms of nasal morbidity. However, the number of patients undergoing the different types of extended approaches was relatively small, making such comparisons potentially misleading. Indeed, the two patients undergoing trancribriform approaches for cribriform plate meningiomata were anosmic pre and postoperatively.

The two groups differ in other ways, apart from the use of the nasoseptal flap: As we started using this approach over the last 3 years, the follow up of such patients is significantly shorter than the follow up of the standard transphenoidal approaches. Indeed, we found that there was a significant positive correlation between elapsed time from surgery and improved QOL, as seen in questions 4 - anosmia, (Spearman Rho. Coefficient - 0.224, $p=0.032)$, question 14 - ear fullness $(0.210, p=0.46)$ and question 22 - Headache $(0.257, p=0.014)$. All of the above tended to improve with time, as seen in Figures 2 and 3. An analysis for other factors associated with poor QOL showed that patient with secreting tumours tended to have much worse quality of life. The difference was statistically significant for Total RSOM score $(p=0.008), Q 11(p=0.007), Q 12(p=$ $0.001), Q 18(p=0.001), Q 19(p=0.024), Q 20(p=0.002), Q 29$ $(p=0.002), Q 30(p=0.008)$ and Q31 ( $p=0.016)$. The globally impaired QOL was most obvious in worse General QOL scores $(p=0.006)$, Emotional QOL scores $(p=0.003)$ and Sleep QOL $(p$ $=0.01)$ Figure 5. Stepwise (0.05 and 0.1$)$ linear regression showed that the only factor associated with worse total RSOM-31 scores was the presence of secreting adenomas.

\section{Discussion}

Quality of life issues

As medicine is adopting a more patient - centered perspective, the use of questionnaires is proving indispensable in the assessment of the impact of disease and of its management: Patient Reported Outcome Measures (PROMs) are measures of health-related quality of life (HRQOL) that are self-rated and reported directly by the patients. They can be broadly divided in to two categories, Generic (measuring some general aspects of quality of life and designed for use in all patient groups or healthy individuals) and Disease - specific (focusing into specific aspects of quality of life and designed for particular conditions or treatments). While generic questionnaire can allow comparison between conditions or treatments, and 


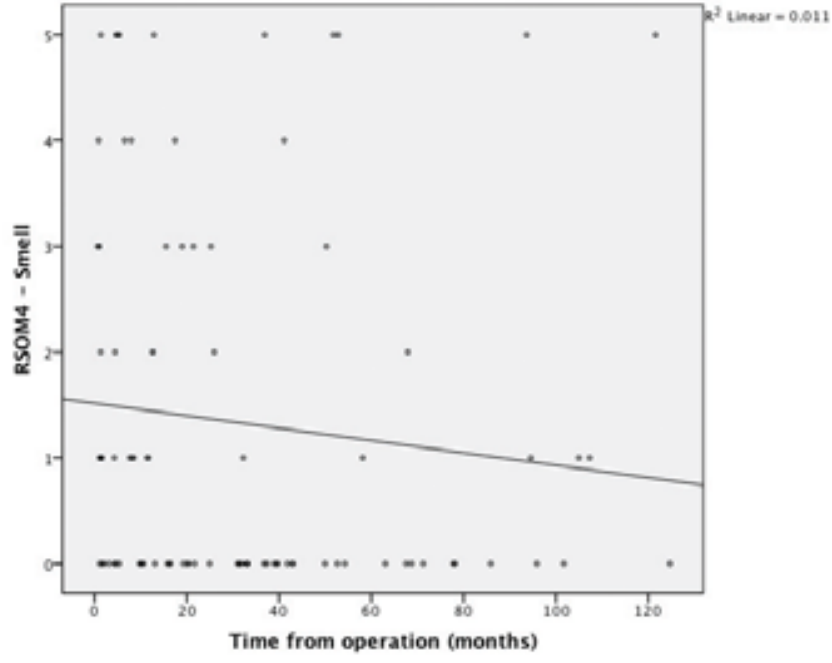

Figure 4. Evolution of hyposmia/anosmia with time from operation.

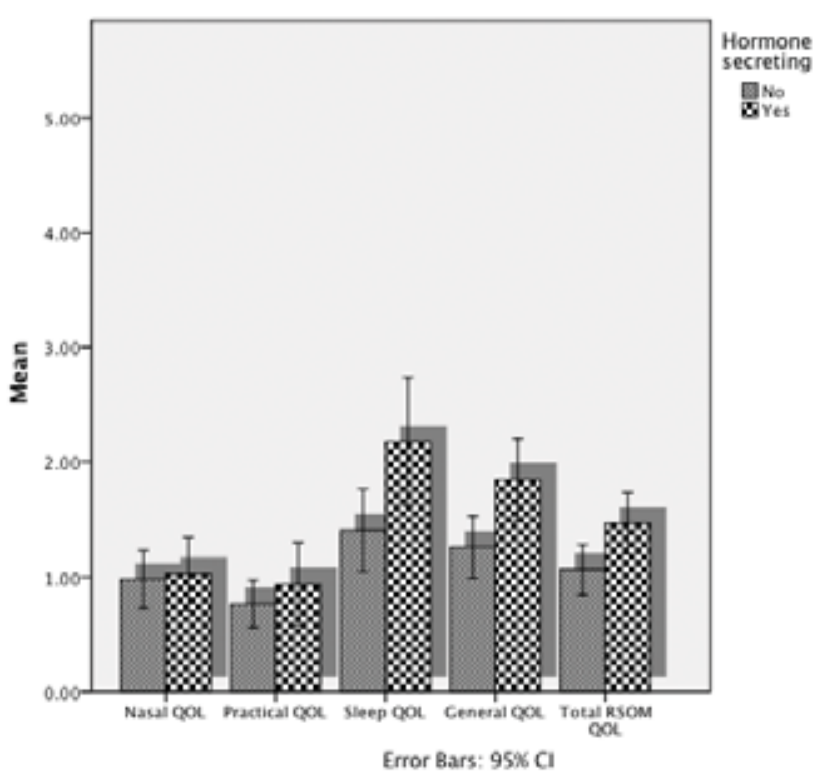

Figure 5. Comparison between Secreting adenomas and remaining tumors (RSOM 31 subscales). therefore can be used to determine the relative cost utility of different interventions and to inform commissioning decisions, they are generally considered less sensitive, especially in rhinological conditions. With that in mind, specific questionnaires have been developed in Rhinology and Skull base surgery. One of the first one to be created was RSOM 31, from which smaller versions (SNOT 22, SNOT 20 and SNOT 16) were derived. The fact the RSOM 31 has been validated ${ }^{(8)}$ and used extensively $(9,10)$ as well as the breadth of the areas it covers were the reasons we used it in our study.

\section{Quality of life and skull base surgery}

Traditionally, most skull basetumours, benign as well as malignant, were removed via subcranial approaches, such as craniofacial resection, involving external craniotomies and extensive facial incisions.

A disease-specific instrument has been designed and validated for those undergoing resection of anterior skull base tumours via external approaches ${ }^{(11)}$. The Anterior Skull Base Questionnaire (ASBQ) instrument includes a total of 35 questions including generic questions, items drawn from head and neck questionnaires, and also includes more detailed questions regarding altered taste, smell, appearance, epiphora, nasal secretions, and visual disturbances. The domains measured include performance, physical function, vitality, pain, specific symptoms, and influence on emotions ${ }^{(12)}$. Using this instrument, 40 patients undergoing subcranial surgery for benign and malignant tumours of the anterior skull base showed a significant deterioration at 6 months after surgery (further exacerbated if the patient underwent radiotherapy) followed by an improvement at 12 months postoperatively ${ }^{(12)}$. However, while this instrument may be useful in patients undergoing external approaches, it has probably limited usefulness in patients undergoing extended endoscopic transnasal approaches, as the potential quality of life issue in such patients undergoing are bound to be different: With that in mind, a more recently developed disease-specific questionnaire focused on endonasal extended approaches on the skull base - the anterior skull base nasal inventory (ASK nasal inventory) ${ }^{(13)}$. However, while useful for assessing nasal mobidity, the 9 questions of this questionnaire refer exclusively to the nose, failing to assess any other aspect of quality of life.

Published studies addressing surgery for pituitary lesions have used global measures, and have identified somewhat reduced quality of life following treatment. Specifically, using the SF 36 there was evidence of a minimal decrease in overall QOL, (in only one-General Health Perception - of the eight domains) ${ }^{(14)}$, while the study by Dekkers et al. ${ }^{(15)}$, assessing 99 patients, 37 of which also underwent radiotherapy, found significant decrease in SF 36 scores in four subscales (social functioning, role limitations due to physical problems, role limitations due to emotional problems, and general health perception). A study from Graham et al., ${ }^{(6)}$ using the RSOM 31 focused on nasal quality of life in 51 patients undergoing transphenoid endo- 
nasal endoscopic removal of pituitary tumours and a historic control of 85 microscopically removed pitiuitary adenomas. He showed overall improved nasal outcomes in the endoscopic group (1.1 versus 1.5 nasal score), however, the response rate of 53 to $59 \%$ renders it potentially open to response bias. The only study until now referring to extended endonasal approaches to the skull base has been the study by Pant et al., ${ }^{(16)}$. This study published in 2010 assessed 51 patients undergoing extended approaches using both the SNOT 22 and the questionnaire developed by Gil: She showed that the quality of life decreased over the first few months to improve later. Unfortunately the lack of data on response rate or the type of tumours removed makes the drawing of conclusions more difficult.

We used RSOM 31 in patients with extended endonasal approaches with nasoseptal flap reconstruction for benign skull base tumours and compared the QOL outcomes with patients undergoing simple endonasal hypophysectomies. The high response rate $(83 \%)$ reduces the response bias and allows us to draw some valid conclusions: We showed that, although most patients have minimal nasal morbidity, there is a price to pay for the use of nasoseptal flap, reflected in statistically significantly worse scores for olfaction and headache. However, three important symptoms, two of which are significantly worse in the extended approach (smell, and headache) improve with time. The fact that smell tends to improve with time has also been shown in the study by Pant et al ${ }^{(17)}$, and we feel that eventually the difference may not be as big as thought. Part of the difference in quality of life must be attributed by the nature of tumours (non-adenomatous tumours or extensive adenomata, often with suprasellar or cavernous sinus extension) dealt via the extensive approach. More importantly, it is fair to assume that most postoperative symptoms tend to improve with time: We feel that the adverse effect of extended approaches would potentially be amplified by the shorter follow up. The fact that we failed to find a clear increase in morbidity, argues against a negative effect of the nasoseptal flap. Indeed, in multivariate regression, the importance of approach reduced further, with the single more important factor determining the quality of life was the presence of a hormone secreting adenoma. We used as a comparison group, patients undergoing standard transphenoidal hypopohysectomy. Both groups had benign skull base tumours, and both were treated via an endonasal approach, with the main difference being in the use of the nasoseptal flap. We feel that this is not optimal, however, it is potentially an effective way to assess the effect of the nasoseptal flap on nasal quality of life per se, something that has not been done before.

We appreciate that a weakness of our study is the lack of preoperative and postoperative data; however, we focused on the nasal symptoms, and we feel that it is fair to assume that much of the postoperative nasal morbidity was caused by the operation rather than by the pathology itself. Another factor that potentially is a weakness is the lack of equal follow-up time for the two groups; indeed, as we embarked in extended approaches in 2007 , the follow-up period is significantly less in this group. However, we factored the amount of follow-up in our analyses, and we feel that in itself, it provides useful information regarding the natural course of postoperative nasal morbidity. While some symptoms do not change significantly with time, others, such as smell and headache, tend clearly to improve with time. It is clear from the questionnaire, that patients describe more severely impaired their Sleep QOL followed by General QOL (fatigue, poor concentration), Emotional QOL (depression, helplessness) with Nasal QOL less impaired.

\section{Conclusion}

Most patients undergoing transnasal endoscopic approaches to the skull base have good nasal quality of life, with nasal complaints low in their overall priorities. Extended approaches with the use of nasoseptal flap appear to have limited negative impact in nasal quality of life, mainly related to headache and reduced smell, both of which improve significantly with time. Hormone-secreting tumours have the most important adverse effect in quality of life extending in general, emotional, sleep and overall wellbeing, as reflected in RSOM 31 subscales.

\section{Author contributions}

CG: designed and planned the study, assisted with collecting the data, did the analysis and wrote the paper. RB: collected most of the data. WvF, SR and WJF: assisted with writing

\section{Conflict of interest}

The authors declare no conflict of interest. 


\section{References}

1. Cappabianca P, Alfieri A, de Divitiis E. Endoscopic endonasal transsphenoidal approach to the sella: towards functional endoscopic pituitary surgery (FEPS). Minim Invasive Neurosurg. 1998; 41: 66-73.

2. Frank G, Pasquini E. Endoscopic endonasal approaches to the cavernous sinus: surgical approaches. Neurosurgery. 2002; 50: 675.

3. Harvey RJ, Shelton W, Timperley D, et al. Using fixed anatomical landmarks in endoscopic skull base surgery. Am J Rhinol Allergy. 2010; 24: 301-305

4. Castelnuovo P, Pistochini A, Locatelli D. Different surgical approaches to the sellar region: focusing on the "two nostrils four hands technique." Rhinology. 2006; 44: 2-7.

5. Hadad G, Rivera-Serrano CM, Bassagaisteguy $\mathrm{LH}$, et al. Anterior pedicle lateral nasal wall flap: a novel technique for the reconstruction of anterior skull base defects. Laryngoscope. 2011; 121: 1606-1610.

6. Rivera-Serrano $\mathrm{CM}$, Oliver $\mathrm{CL}$, Sok J, et al. Pedicled facial buccinator (FAB) flap: a new flap for reconstruction of skull base defects. Laryngoscope. 2010; 120: 1922-1930.

7. Hadad G, Bassagasteguy L, Carrau RL, et al. A novel reconstructive technique after endoscopic expanded endonasal approaches: vascular pedicle nasoseptal flap. Laryngoscope. 2006; 116: 1882-1886.
8. Zanation AM, Carrau RL, Snyderman $\mathrm{CH}$, et al. Nasoseptal flap reconstruction of high flow intraoperative cerebral spinal fluid leaks during endoscopic skull base surgery. Am J Rhinol Allergy. 2009; 23: 518-521.

9. Piccirillo JF, Edwards D, Haiduk A, Yonan C, Thawley SE. Psychometric and Clinimetric Validity of the 31-Item Rhinosinusitis Outcome Measure (RSOM-31). Am J Rhinology. 1995; 9: 297-306.

10. Javer AR, Genoway KA. Patient quality of life improvements with and without computer assistance in sinus surgery: outcomes study. J Otolaryngol. 2006; 35: 373-379.

11. Georgalas C, Hansen F, Videler WJM, Fokkens WJ. Long terms results of Draf 3 procedure. Rhinology. 2011; 49: 195-201.

12. Abergel A, Fliss DM, Margalit N, Gil Z. A prospective evaluation of short-term healthrelated quality of life in patients undergoing anterior skull base surgery. Skull Base. 2010; 20: 27-33.

13. Little AS, Jahnke $H$, Nakaji $P$, et al. The anterior skull base nasal inventory (ASK nasal inventory): a clinical tool for evaluating rhinological outcomes after endonasal surgery for pituitary and cranial base lesions. Pituitary. 2011. Available at: http://www. ncbi.nlm.nih.gov/pubmed/22038032. Accessed January 3, 2012.

14. Karabatsou K, O'Kelly C, Ganna A, Dehdashti
AR, Gentili F. Outcomes and quality of life assessment in patients undergoing endoscopic surgery for pituitary adenomas. $\mathrm{Br} \mathrm{J}$ Neurosurg. 2008; 22: 630-635.

15. Dekkers OM, van der Klaauw AA, Pereira AM, et al. Quality of life is decreased after treatment for nonfunctioning pituitary macroadenoma. J Clin Endocrinol Metab. 2006; 91: 3364-3369.

16. Graham SM, Iseli TA, Karnell LH, et al. Endoscopic approach for pituitary surgery improves rhinologic outcomes. Ann Otol Rhinol Laryngol. 2009; 118: 630-635.

17. Pant $H$, Bhatki AM, Snyderman $\mathrm{CH}$, et al. Quality of life following endonasal skull base surgery. Skull Base. 2010; 20: 35-40.

\section{Christos Georgalas}

Director, Endoscopic Skull Base

Centre - A2-228

Department of Otolaryngology

Academic Medical Centre

Meibergdreef 9

1105AZ Amsterdam

The Netherlands 